\title{
GLOBAL BIFURCATIONAL APPROACH TO THE ONSET OF SPATIO-TEMPORAL CHAOS IN REACTION DIFFUSION SYSTEMS
}

\author{
YASUMASA NISHIURA*
}

\begin{abstract}
A new criterion for the onset of spatio-temporal chaos arising in the Gray-Scott model is presented. This is based on the interrelationship of global bifurcating branches of stationary or periodic solutions with respect to the removal rate contained in the model, especially their locations of saddle-node points and the Hopf bifurcation point of a constant state play a key role. At the onset point there exists a generalized heteroclinic cycle on the whole line and spatio-temporal chaos emerges by unfolding this cycle.
\end{abstract}

1. Introduction. Since early 90 's, a variety of chemical patterns have been observed in chemical laboratories ([7], [13], [14]), and among them, self-replicating patterns (SRP) and spatio-temporal chaos (STC) are spectacular examples. Selfreplicating patterns have been also observed numerically in a reaction diffusion system ([1], [5], [8], [9]). Several interesting analytical works have also appeared recently: For instance, construction of single-spot solution to the Gray-Scott model (see below) and its stability has been done by [6] with the aid of formal matched asymptotic analysis, which is closely related to the splitting phenomenon; A rigorous analysis concerning the existence and stability of steady single pulse as well as nonexistence of traveling pulses has been done quite recently by [10] and [11]. Although we have found a nice collection of exciting dynamics, we don't know yet how and why the transitions occur from one dynamics to another, especially what kind of mathematical mechanism causes STC. The main issue is to understand the dynamic phase transition of the following Gray-Scott model ([15]) from global bifurcational view point.

$$
\left\{\begin{array}{l}
\frac{\partial u}{\partial t}=D_{u} \nabla^{2} u-u v^{2}+F(1-u) \\
\frac{\partial v}{\partial t}=D_{v} \nabla^{2} v+u v^{2}-(F+k) v
\end{array}\right.
$$

where $u$ and $v$ are concentrations of the chemical materials $U$ and $V$, respectively, $D_{u}$ and $D_{v}$ the diffusion coefficients, $F$ the in-flow rate of $U$ from outside, $F+k$ the removal rate of $v$ from reaction field. The main feature of the associated ODE of (1.1) has a Bogdanov-Takens (BT)-point together with a stable critical point $(1,0)$. BT-point is a singularity of codim 2 where saddle-node and Hopf bifurcations merge there in 2-dimensional parameter space $(k, F)$ (see Fig. 1.1). Before the saddle-node bifurcation, $(1,0)$ is the only equilibrium point and globaly stable, and hence the system is of excitable character. We denote the two constant states born from the saddle-node bifurcation by $P$ and $Q$. When $k>0.035$, the excitable character persists until the recovery of stability of the equilibrium point $P$ due to the Hopf bifurcation, since the newly born critical points $P$ and $Q$ are unstable and there are no stable periodic orbits owing to the subcriticality of the Hopf bifurcation (see Fig. 1.1).

The aim of this paper is of two-fold. One is to clarify the onset point of spatiotemporal chaos arising in the Gray-Scott model. A global bifurcational diagram of

*Laboratory of Nonlinear Studies and Computations, Research Institute for Electronic Science, Hokkaido University, Kita-ku, Sapporo, 060-0812, Japan (nishiura@aurora.es.hokudai.ac.jp). This research was supported in part by Grant-in-Aid for Scientific Research 11214201, Ministry of Education, Science and Culture, Japan, and 11874032, JSPS. 

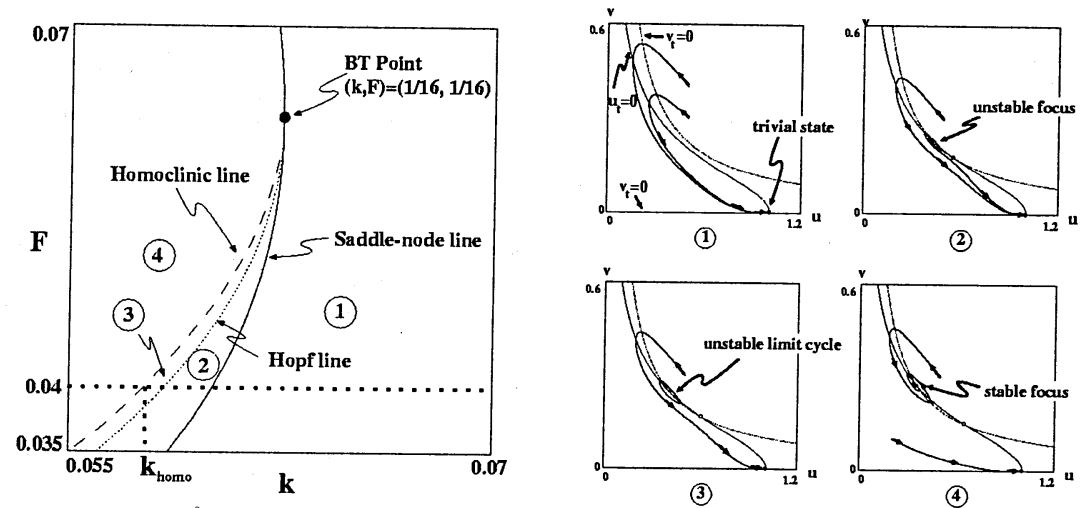

FIG. 1.1. Unfolding of BT-point and flows of the kinetics for the GS-model. BT-point is a codim 2 singularity where fold and Hopf bifurcations merge there in $(k, F)$-space. Solid (resp.dotted) line represents fold (resp.Hopf)bifurcation. Unfolding BT-point, there appears a Homoclinic line as indicated. The flows in the phase space are computed at $F=0.04$.

ordered states plays a key role to give such an criterion. The other is to understand the detailed mechanism which drives a chaotic behavior from a pattern formation point of view, namely we introduce four elementary basic dynamics, then put them together in an aftereffect region of parameter space. The loose meaning of aftereffect is a kind of memory of saddle-node bifurcation point, namely if one takes a parameter value close to the saddle-node (SN) point and chooses an initial data close to the solution at the SN point, then the orbit stays there for certain time and its dynamical behavior is close to that of the $\mathrm{SN}$ point.

In other words we would like to understand the spatio-temporal chaos (STC) in a constructive way from a pattern formation view point rather than projecting the infinite dimensional dynamics to an effective finite dimensional one.

There are three different types of STC's for the Gray-Scott model depending on parameter values. These three STC's are classified according to the constituting members of basic dynamics (for the list of the basic dynamics, see Section 3.1). Fig. 1.2(a)(resp. Fig. 1.2(b)) is called STC of static (resp. traveling type) type, since the quasi-ordered state observed during the process is steady state (resp. traveling pulse). On the other hand, Fig. 1.2(c) has a different character, namely it has an annihilation process when two pulse waves collide. In this report we only treat the non-annihilation case.

It should be noted that STC is observed only in a restricted region in the parameter space (see region 4 in Fig. 2.1), in fact, STC of static type, for instance, disappears when the parameter $k$ is increased, and is replaced by convergence to a periodic stationary pattern through self-replication dynamics. The main issue here is therefore to clarify what kind of mathematical structure controls the transition from ordered state to STC. Finally this work is based on the joint work with Daishin Ueyama and the more details will be reported elsewhere.

\section{Self-replication and self-destruction.}

2.1. Phase diagram of PDE dynamics in 1D. In order to obtain a nontrivial pattern from the background state $(1,0)$, it is necessary to add a perturbation of finite amplitude to it, since $(1,0)$ is locally stable in PDE sense. In fact starting from a spiky localized initial data, the Gray-Scott model displays a variety of dynamics 


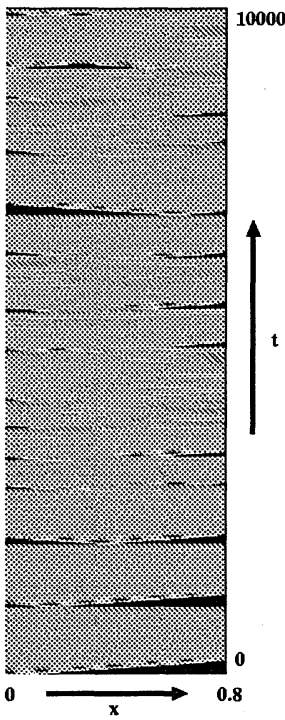

(a)

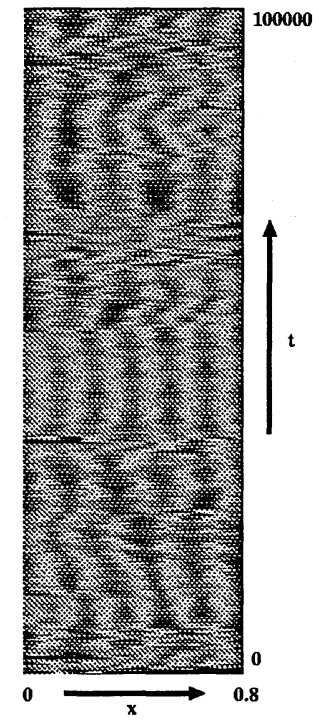

(b)

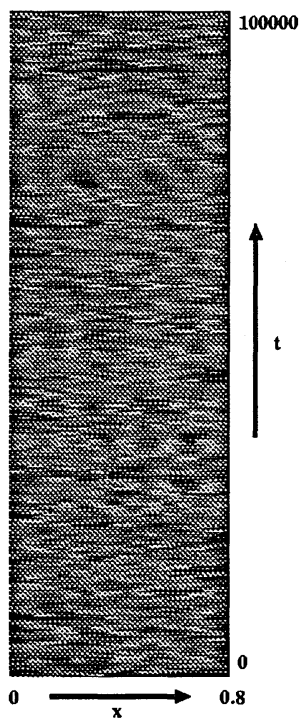

(c)

Fig. 1.2. Three different types of spatio-temporal chaos. $(a) F=0.035,(b) F=0.02,(c) F=0.015$

as in Fig. 2.1. There are at least five different regimes in $(k, F)$-space: standing pulse, traveling pulse, self-replication patterns, traveling front, and spatio-temporal chaos, respectively. Each transition from one regime to another is sharp and the main objective is to clarify the mechanisms of such transitions, especially to chaotic one. It turns out that a global interrelation among the branches of the ordered states becomes important, in particular, the relation between locations of saddle-node points of them and the Hopf bifurcation point of the constant state $P$ becomes crucial to give a criterion for such a transition.

2.2. Self-replication. Transition from standing wave or traveling wave to selfreplicating pattern was clarified in [2]. The essence is that all the ordered patterns disappear due to the saddle-node structures almost simultaneously, but the strong (fading) memory remains in the phase space if the parameter is close to the location of the saddle-node bifurcation. This aftereffect and the connections among saddlenode branches are key ingredients to understand the self- replication dynamics. For details, see [2].

2.3. Self-destruction. Very simple, but an important example of the aftereffect of saddle-node point is a self-destruction. We already saw an example for the FitzHugh-Nagumo equations in the previous section. Suppose there exists a saddlenode branch of an ordered state, say a stationary pattern as in Fig. 2.2 and the associated unstable manifold is connnected to an homogeneous state. Then the corresponding aftereffect displays a self-destruction, namely an ordered state persists for a while, the duration of which depends on how close the parameter value to the saddlenode bifurcation point, but eventually decays to the constant state. It is possible to construct an invariant manifold near the saddle-node point and derive an ODE on it (see [4] ), and it turns out that the duration time of the aftereffect is of order $O\left(\epsilon^{-1 / 2}\right)$ where $\epsilon$ denotes the distance from the saddle-node point in the parameter space.

The above examples look so simple, however the whole dynamics becomes more 

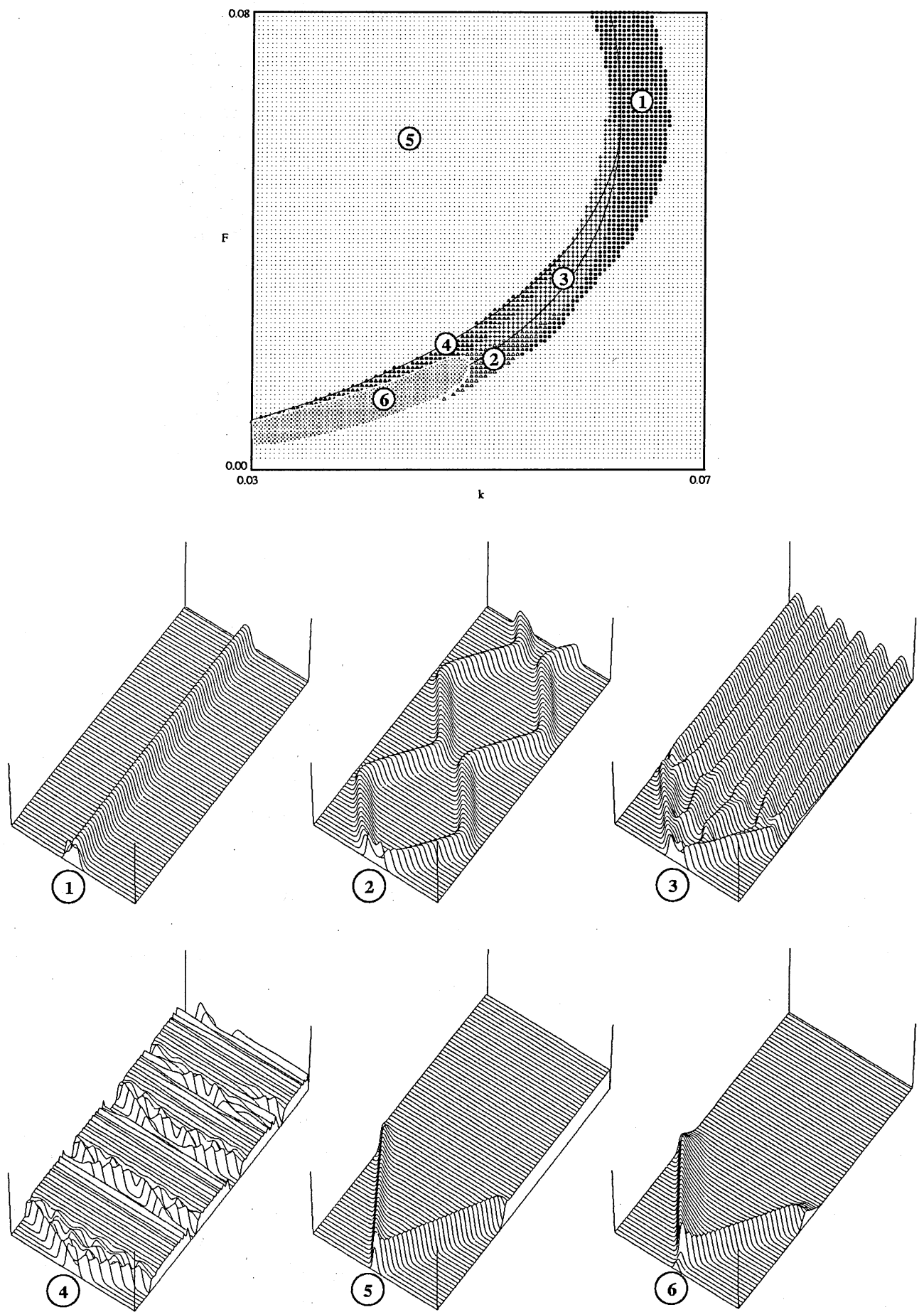

FIG. 2.1. The phase diagram for 1D. The initial condition is a localized step function and the boundary condition is of Neumann type. Region 1: Standing pulse, Region 2: Traveling pulse, Region 3: Self-replicating patterns, Region 4: Spatio-temporal chaos, Region 5: Traveling front, Region 6: Annihilation. 


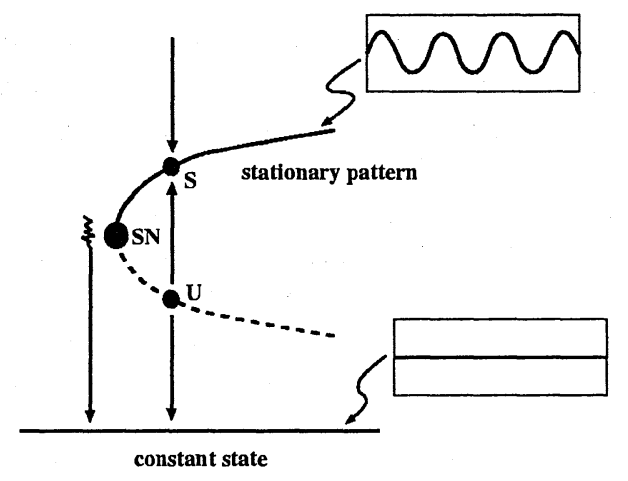

FIG. 2.2.

exotic and complicated when they are combined with other dynamics, which we will see in the next section.

3. Spatio-temporal chaos. There are many routes to chaos like perioddoubling, breakdown of torus, intermittency and so on. As we saw in Section 1, there are several types of spatio-temporal chaos arising in the Gray-Scott model. The non-annihilation case maybe classified as an intermittency type I (see, for instance, [3]), however, from pattern formation point of view, this does not explain much about the detailed mechanism of pattern dynamics, for instance, why the reinjection occurs for the non-annihialtion case in the infinite-dimensional space. Somehow we would like to understand the geometric structure of spatio-temporal chaos for the Gray-Scott model like the Shilnikov mapping in finite dimensional space.

In this section we first present a new criterion for the onset of spatio-temporal chaos, which is based on an interrelationship among global bifurcation branches of ordered states and its influence over the dynamics as an aftereffect. Second, by taking this view point, we can understand the detailed mechanism of the spatio-temporal chaos from pattern formation view point without projecting the whole dynamics to an effective finite dimensional space. In fact we find basic building blocks of pattern dynamics participating in the spatio-temporal chaos, and the above view point allows us to know how each block contributes to form chaotic behavior. It turns out that there are at least two different basic mechanisms, which are responsible for the onset of STC, depending on whether annihilation occurs or not. One is a heteroclinic cycle and its unfolding in infinite dimensional space, and the other is creation (by spliting) and destruction (by annihilation) process. In this paper, we! focus on the non-annihilation case. The annihilation case will be discussed in a forthcoming paper. When a parameter, say $k$, is continuously changed, the onset of STC seems to occur abruptly as far as we only look at ODE dynamics and the local bifurcations. This reflects the fact that such a transition is caused by some global structure far from equilibrium. It turns out that such an abruptness can be resolved into a natural consequence, once we employ a global bifurcation view point.

3.1. Pattern-switching processes and heteroclinic cycles. To understand the STC dynamics from pattern formation point of view, it is necessary to clarify what kind of basic pattern dynamics participates in forming STC. There are four basic dynamics which switch one state to another. 
1. ODE dynamics: Switching from the homogeneous state $P$ to the background state $(1,0)$. There exists a $k=k_{\text {Hopf }}$ such that $P$ is unstable for $k>$ $k_{H o p f}$ and the homogeneous limit cycle emanating at $k=k_{\text {Hopf }}$ is unstable and exists only for $k<k_{\text {Hopf }}$ (subcritical), hence $P$ is taken over by the background state $(1,0)$ by homogeneous perturbation.

2. SRP dynamics: Switching from the backgroud state to an ordered structure. This is brought by SRP dynamics of Section 2.2, i.e., when a localized perturbation of finite amplitude is added to $(1,0)$, then modulating front (or SRP of front type) invades into $(1,0)$ and spatially periodic structure is formed after the front.

3. Self-destruction: Switching from an ordered structure to the homogeneous state $P$. This is the destruction process explained in Section 3.3, i.e., aftereffect of the unstable manifold connecting stationary periodic pattern to the homogeneous state.

4. Annihilation: Switching from an ordered state to the background state by annihilation. This is observed in the annihilation regime in $(k, F)$-space where two traveling pulses disappear by head-on collision.

The first three processes can be regarded as heteroclinic orbits on a whole line, for instance, the second one is a heteroclinic orbit connecting the constant state $(1,0)$ to a spatially periodic pattern as in Fig. 3.1.

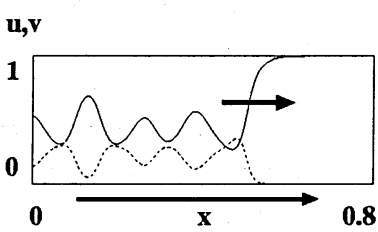

(a)

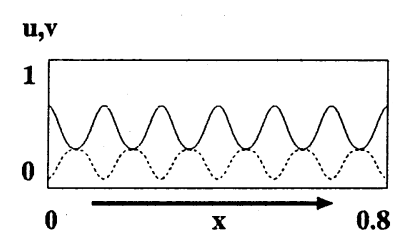

(b)

FIG. 3.1. $(F=0.035, k=0.0566, L=0.8)$

Moreover it should be noted that these three connections constitute formally a heteroclinic cycle on $\mathbf{R}$ provided that all the connections occur exactly at the same parameter value. Such an miraculous coincidence does not seem to occur generically, however it turns out that such a delicate tuning is controllable by looking at the parametric dependency of global branches, and a trigger necessary to provoke a front is spontaneously formed due to the non-uniform (in space) destruction to $P$. Here we loosely describe how an orbit makes a cycle starting from the unstable constant state $P$ through three switching processes (see Fig. 3.2). Suppose a small perturbation of long wavelength is added to $P$, initial phase difference is amplified and the solution goes to $(1,0)$ on phase-gaining part of the interval and the remaining part, which is still far from $(1,0)$, becomes a trigger of invading front into $(1,0)$ followd by an periodic stationary ordered pattern. Finally this ordered state decyas to the homogeneous state $P$ through the destruction process. This completes the cycle. Strictly speaking, the terminology heteroclinic cycle is not appropriate, since, as we will see in the next section, the spatially periodic pattern is not hyperbolic, however we use this terminology in a generalized sense in the sequel.

3.2. Unfolding the heteroclinic cycle - spatio-temporal chaos for nonannihilation case -. Each process of dynamics in the previous section does not show any chaotic behavior by itself, however if they are combined together appropriately, 


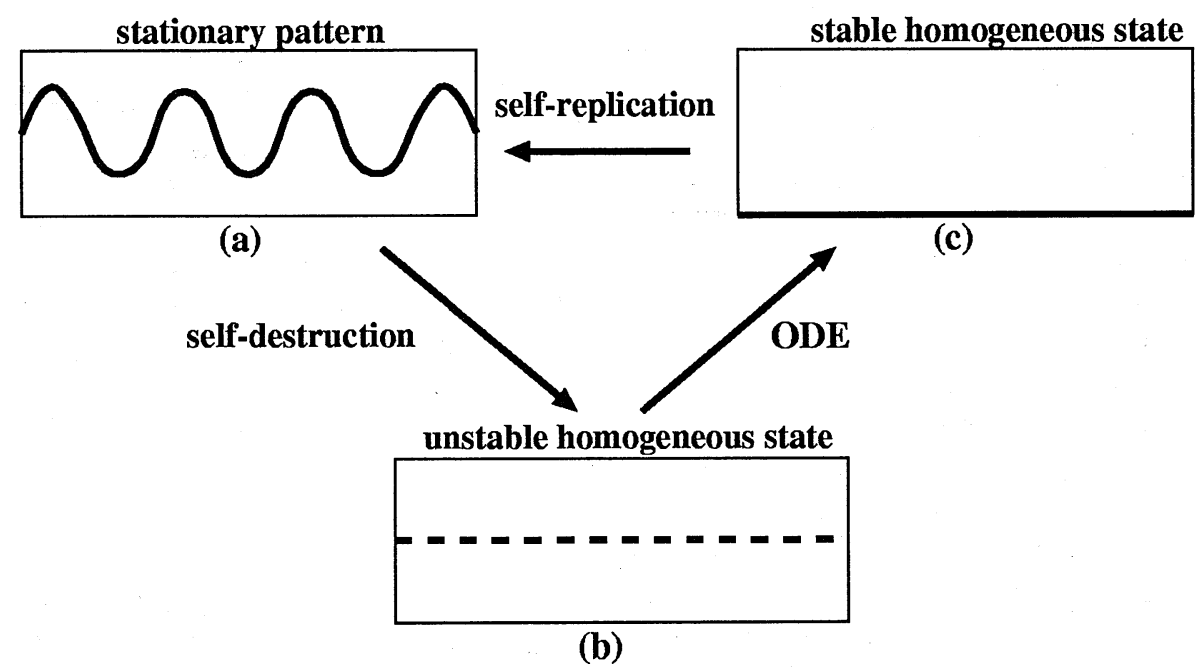

FIG. 3.2 .

the resulting one becomes chaotic in space and time. The main idea is to unfold the heteroclinic cycle in infinite dimensional space by adjusting the parameters in such a way that the orbit itinerate this cycle chaotically in space and time. This is apparently a reminiscent of the homoclinic tangling in finite dimensional space. Here the concept aftereffect again becomes useful to achieve such an itinerancy, in fact, it is absolutely necessary to consider all the basic dynamics in an aftereffect regime, otherwise the orbit is stuck somewhere and does not itinerate. The existence of a parameter region where such an chaotic itinerancy occcurs strongly depends on the global interrelationship of ordered and homogeneous branches, which we shall discuss more precisely in the next subsection. In particular heteroclinicity heavily depends on the stability property of the homogeneous state $P$, in fact, if $P$ is repelling, it becomes a driving force which together with the self-destruction dynamics sends some part (in space) of the solution to the background state without annihilation. Surprisingly the solution orbit approaches the unstable $P$ locally in space even if the orbit starts from an initial data of step-function. This is due to the fact that $P$ loses its stability in ODE sense, i.e., in constant space, and the pattern born after the front is almost average zero with respect to $P$, so it lies close to the stable manifold of $P$. When the orbit becomes very close to $P$, the instability becomes dominant and it starts to oscillate and eventually goes to the background state $(1,0)$. Non-uniformity of the convergence to $(1,0)$ in space is apparently quite important to form a heteroclinic cycle. If the system size is small, then the orbit first becomes almost homogeneous close to $P$ then settles down to $(1,0)$. More precisely there should be a balance among several time scales not to have a synchronization in space, namely the strength of diffusivity (= system size), velocity of the front, instability of $P$, and the strength of the aftereffect (= how far $k$ is located from the saddle-node point), although these are related each other.

3.2.1. STC of static type. We shall discuss how the the dynamics is controlled by the global arrangement of branches. STC of this type is typically observed in the area 4 of Fig. 2.1 and a typical time-evolution at $F=0.035$ and $k=0.05632$ is given by Fig. 1.2(a). On the other hand, for slightly larger $F$, say $F=0.04$, we do not 
observe any chaotic pattern. To understand this difference and the onset of STC, we draw two global bifurcation diagrams of stationary patterns for each $F$ (see Fig. 3.3). The main difference of two diagrams (a)(left) and (b)(right) of Fig. 3.3 is the order of two quantities $k_{\min }$ and $k_{\text {Hopf }}$, namely $k_{\min }<k_{\text {Hopf }}$ for (a) and $k_{\min }>k_{\text {Hopf }}$ for (b). Here the $k_{\text {min }}$ is the smallest value of $k$ among the locations of saddle-node points of the ordered states. $k_{m i n}$ usually, as in Fig. 3.3, coincide with the SN-point of stationary branch which is the continuation of the final state after self-replication, and the $k_{\text {Hopf }}$ is the Hopf bifurcation point of the homogeneous state $P$. The $k_{H o p f}-$ line is explicitly calculated and becomes a monotone function of $F$ (see Fig. 3.5). The $k_{m i n}$-line is much harder to compute, since it is a locus of saddle-node points of stationary branches depending on $F$. It turns out that the function $k_{\text {min }}(F)$ is also monotone, but less steap than $k_{\text {Hopf }}(F)$ (see Fig. 3.5). Therefore there exists a unique intersecting point $G$ in $(k, F)$-space as in Fig. 3.5, and the order of these quantities are reversed at $G$. It should be noted that the curve $k_{\min }(F)$ perfectly fits the right-hand side of the boundary of STC region 4 in Fig. 2.1, which reveals the nature of the transition boundary.
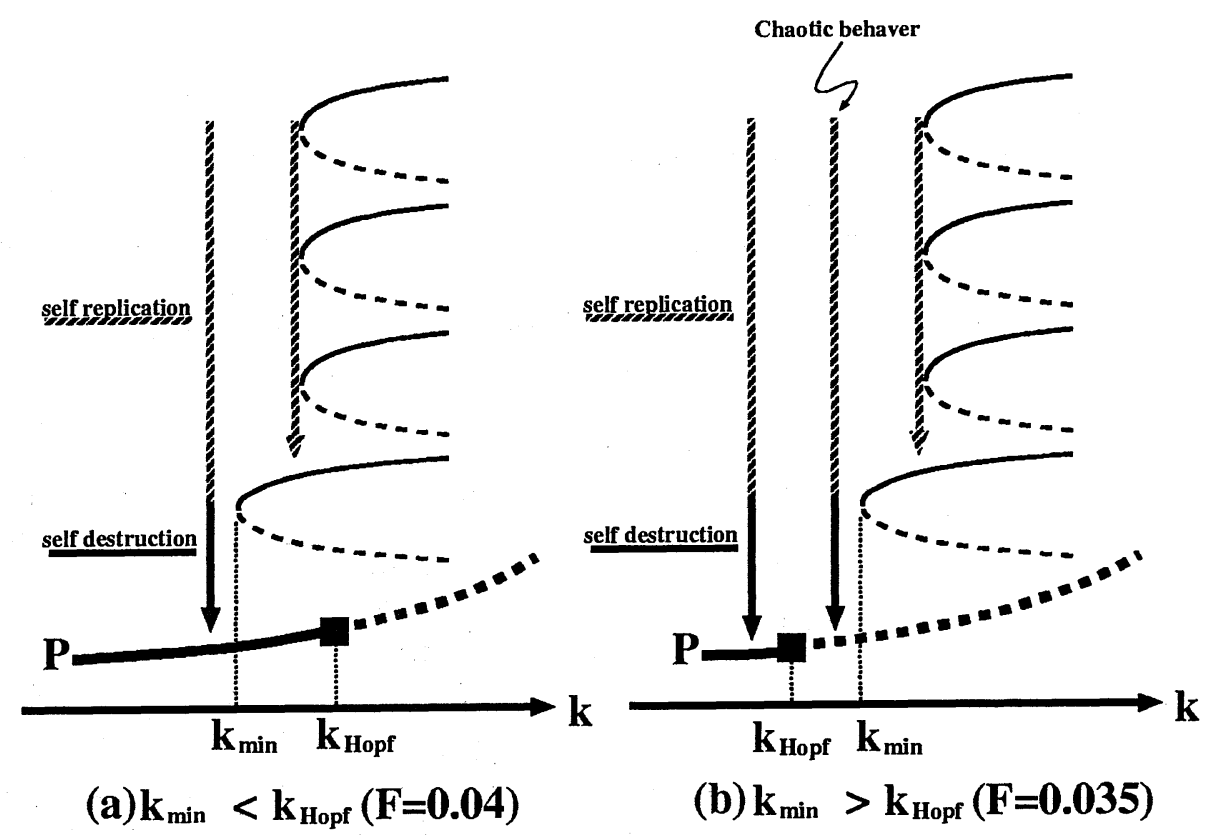

FIG. 3.3. (a)Left: The schematic bifurcation diagram for $F=0.04$. After self-destruction, the orbit settles down to the stable homogeneous state. (b)Right: The schematic bifurcation diagram for $F=0.035$. Since the homogeneous state loses its stability due to Hopf bifurcation, the orbit itinerates in a chaotic way.

The region below $G$ sandwitched by two curves $k_{\text {min }}$ and $k_{\text {Hopf }}$ is called $S T C$ (spatio-temporal chaos) region where we observe spatio-temporal chaos. The bifurcation diagram in this regime is given by Fig. 3.4.

Heteroclinic cycle can be constructed at least formally at any point on the right boundary of STC-region (i.e., $k=k_{\min }(F)$ ), and STC is observed as a result of unfolding of this cycle. Heteroclinic cycle at $k=k_{\text {min }}$ consists of three parts (see Fig. 3.2$): P$ to $(1,0),(1,0)$ to stationary pattern, and the destruction process to $P$. As remarked before, the above stationary pattern is not hyperbolic, however 


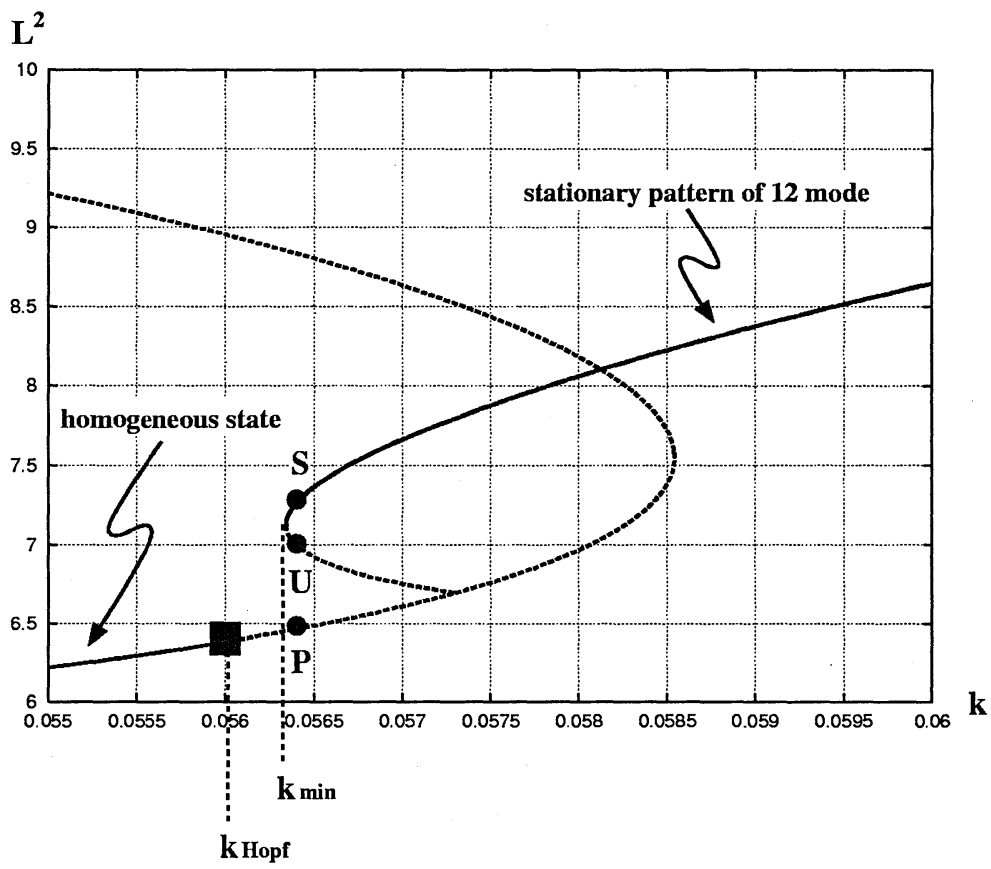

FIG. 3.4 .

we use "heteroclinic" in a generaized sense. Note that the connection between the unstable part of the saddle-node branch and the homogeneous state $P$ still persists even if $P$ loses its stability. When $k$ belongs to the interval $k_{H o p f}<k<k_{\text {min }}$ (see Fig. 3.3(b)), the spatio-temporal chaos is invoked in the following way. A spatially periodic pattern is formed after the SRP process initiated by the initial trigger, but it only lasts for a while and approaches $P$ due to the aftereffect of the connection to $P$. Since $P$ is unstable, the orbit bounces except that it accidentally lies on the stable manifold of $P$. Recalling that the intial data is a localized spiky perturbation of finite amplitude, the process of approaching $P$ does not occur uniformly on the interval $I$ as is shown in Fig. 1.2; the solution is close to $P$ on some portion of the interval, hence ODE dynamics becomes dominant there, and therefore it reaches quickly background state. Once such an background region is formed, the SRP wave (or modulating front) starts to propagates into this region and $(1,0)$-state is taken over by a periodic structure, however the periodic structure after the front survive only temporalily in this parameter regime as before, it eventually disappear and becomes close to homogeneous state $P$ again. In this manner the orbit does not settle down to any ordered state and makes a chaotic cycle. On the other hand, if $F$ is above $G$ (for instance $F=0.04$ ), the order of two quantities $k_{m i n}$ and $k_{H o p f}$ is reversed as in Fig. 3.3(a). In this case self-destruction process also occurs for $k<k_{\min }$, however no heteroclinic cycle is formed since $P$ is stable, we have instead a traveling front connecting $(1,0)$ to $P$ like region 5 of Fig. 2.1. A similar thing also happens for the case Fig. 3.3(b), if $k$ is chosen to be smaller than $k_{H o p f}$. In view of the above discussion, the intersecting point 


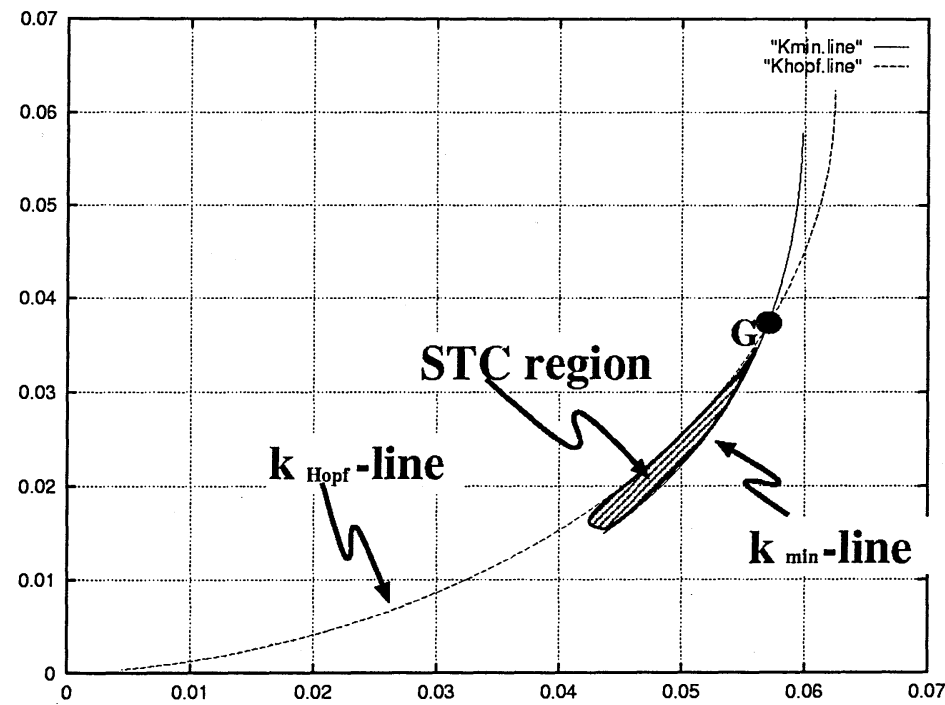

(a)

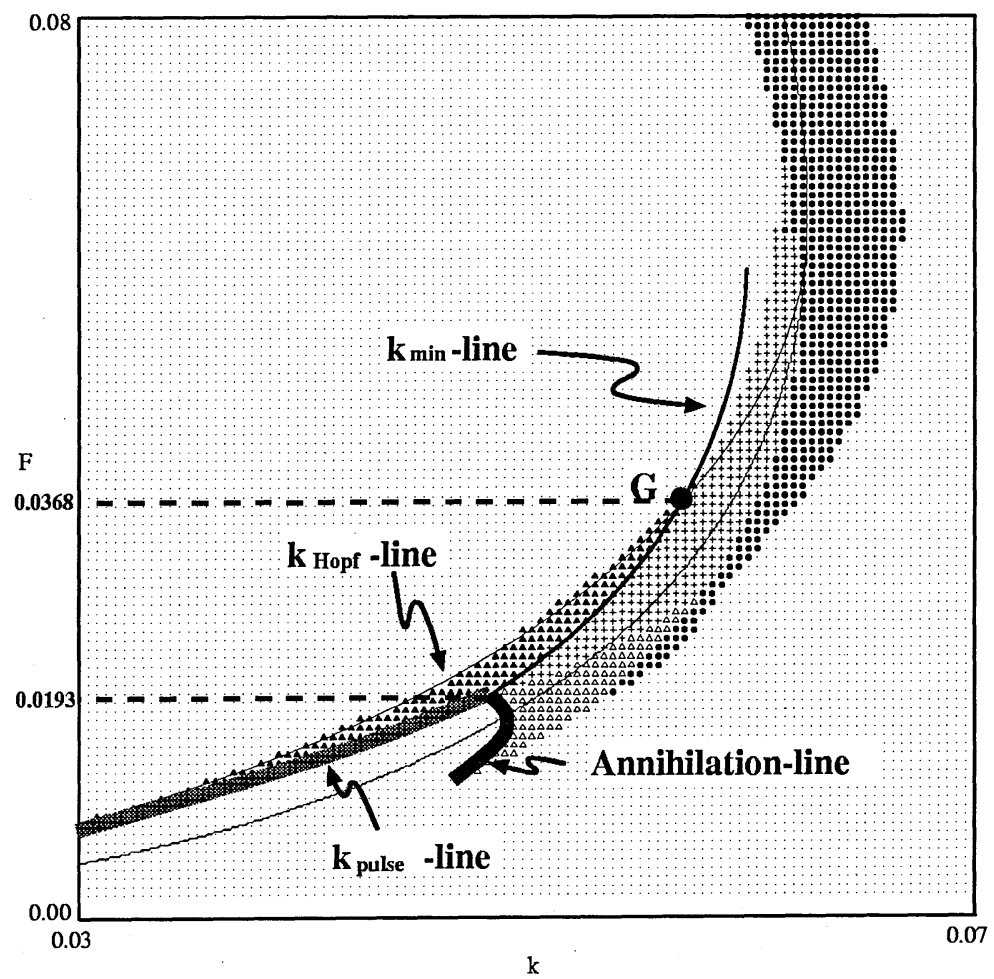

(b)

FIG. 3.5 . 
gives the onset point $G$ of spatio-temporal chaos in the phase diagram Fig. 2.1, and the boundaries of STC region (black triangle region) consists of two curves $k=$ $k_{\text {min }}(F)$ (lower boundary) and $k=k_{\text {Hopf }}(F)$ (upper boundary). We conclude that STC region of static type is completely characterized by two quantities $k_{m i n}$ and $k_{\text {Hopf }}$.

\section{REFERENCES}

[1] J. E. Pearson, Complex patterns in a simple system, Science Vol., 216 (1993), pp. 189-192.

[2] Y. Nishiura AND D. UEYAMA, A skeleton structure of self-replicating dynamics, Physica D, 130 (1999), pp. 73-104.

[3] E. Отто, Chaos in dynamical systems, Cambridge Univ.Press (1993).

[4] S. EI, Y. Nishiura AND B. SANDSTEDe, Pulse interaction approach to self-replicating dynamics in reaction diffusion systems, preprint 1999.

[5] W. N. ReYnolds, J. E. PEARSON AND S. PONCE-DAWSON, Dynamics of self-replicating patterns in reaction diffusion systems, Physical Review Letters, Vol.72, 17 (1994), pp. 1120-1123.

[6] W. N. Reynolds, S. Ponce-Dawson and J. E. Pearson, Self-replicating spots in reactiondiffusion systems, Physical Review E, Vol.56, 1 (1997), pp. 185-198.

[7] P. De Kepper, J. J. Perraud, B. Rudovics and E. Dulos, Experimental study of stationary turing patterns and their interaction with traveling waves in a chemical system, International Journal of Bifurcation and Chaos, Vol.4, 5 (1994), pp. 1215-1231.

[8] K. E. Rasmussen, W. Mazin, E. Mosekilde, G. Dewel and P. Borckmans, Wave-splitting in the bistable Gray-Scott model, International Journal of Bifurcation and Chaos, Vol.6, 6 (1996), pp. 1077-1092.

[9] V. PETRov, S. K. SCOTT AND K. Showalter, Excitability, wave reflection, and wave splitting in a cubic autocatalysis reaction-diffusion system, Phil. Trans. Roy. Soc. Lond. A, 347 (1994), pp. 631-642.

[10] A. Doelman, T. J. Kaper And P. A. Zegeling, Pattern formation in the one-dimensional Gray-Scott model, Nonlinearity, 10 (1997), pp. 523-563.

[11] A. Doelman, R. A. Gardner AND T. J. KAPER, Stability analysis of singular patterns in the 1-D Gray-Scott model: A matched asymptotic approach, preprint.

[12] A. Doelman, W. ECKhaus AND T. J. KAPER, Slowly-modulated two pulse solutions and pulse splitting bifurcations, preprint.

[13] K. J. LeE, W. D. McCormick, J. E. PeArson And H. L. Swinney, Experimental observation of self-replicating spots in a reaction-diffusion system, Nature, 369 (1994), pp. 215-218.

[14] K. J. LEE AND H. L. SWINNEY, Lamellar structures and self-replicating spots in a reactiondiffusion system, Phys.Rev.E, 51 (1995), pp. 1899-1915.

[15] P. GRAY AND S. K. ScotT, Autocatalytic reactions in the isothermal, continuous stirred tank reactor: oscillations and instabilities in the system $A+2 B \rightarrow 3 B, B \rightarrow C$, Chem. Eng. Sci., 39 (1984), pp. 1087-1097. 
Y. NISHIURA 